NOTA

\title{
Nuevo registro del huroncito patagónico (Lyncodon patagonicus, Carnivora: Mustelidae] para el centro de Argentina y aportes sobre su historia natural
}

\author{
New record of the Patagonian weasel (Lyncodon patagonicus, \\ Carnivora: Mustelidae] for the center of Argentina and \\ contributions about its natural history
}

D.O.I.: https://doi.org/10.30550/j.azl/2018.62.1/4

\author{
Guillermo Sferco ${ }^{1}$, Martín Toledo ${ }^{1,2}$, Elvira Canio ${ }^{1,2}$, Agustina Tello ${ }^{1,2}$, \\ Adrián Giaquinta ${ }^{2}$, Florencia Frola Mendizabal $^{2}$, Florencia Rossi ${ }^{2}$ \\ 1 Centro de Zoología Aplicada, Facultad de Ciencias Exactas, Físicas y Naturales, Universidad Nacional \\ de Córdoba. Córdoba, Argentina. Correo electrónico: g.sferco@unc.edu.ar \\ a Facultad de Ciencias Exactas, Físicas y Naturales, Universidad Nacional de Córdoba. Córdoba, Argen- \\ tina.
}

> Resumen - El huroncito patagónico (Lyncodon patagonicus) habita regiones áridas y semiáridas de Argentina y Chile. La mayor cantidad de referencias proviene de registros antiguos, existiendo escasas observaciones recientes. Si bien la especie es conocida desde hace más de un siglo y posee una amplia distribución, el conocimiento sobre su historia natural es limitado. En este trabajo se da a conocer una nueva localidad para esta especie en base a un ejemplar observado y fotografiado en la Reserva Natural Chancaní, provincia de Córdoba, Argentina. Además aportamos información sobre su historia natural, ya que el individuo fue visto durante el día, alimentándose de un ejemplar de Ctenomys sp. Por lo tanto, confirmamos la presencia del huroncito patagónico en la provincia de Córdoba, cuyo registro más fehaciente data del Pleistoceno Tardío.

Palabras clave: Alimentación, Argentina, distribución, Reserva Chancaní.

> Abstract - The Patagonian weasel (Lyncodon patagonicus) inhabits arid and semiarid regions of Argentina and Chile. Most references come from ancient records, recent observations are scarce. Although the species has been known for more than a century and has a wide distribution, knowledge about its natural history is limited. In this work, a new locality is documented for this species, based on a single specimen observed and photographed at Chancaní Natural Reserve, Córdoba Province, Argentina. It also provides information about its natural history, since the individual was seen feeding on a specimen of Ctenomys sp. during the day. Therefore, we confirm the presence of the Patagonian Weasel in the province of Córdoba, whose most reliable record of the species comes from Late Pleistocene.

Keywords: Argentina, Chancaní Reserve, distribution, feeding.

\footnotetext{
> Ref. bibliográfica: Sferco, G., Toledo, M., Canio, E., Tello, A., Giaquinta, A., Mendizábal, F. F., Rossi, F. (2018). Nuevo registro del huroncito patagónico (Lyncodon patagonicus, Carnivora: Mustelidae) para el centro de Argentina y aportes sobre su historia natural. Acta zoológica lilloana 62 (1): 36-41.

> Recibido: 07/03/18 - Aceptado: 09/05/18

$>$ URL de la revista: http://actazoologica.lillo.org.ar 
El huroncito patagónico Lyncodon patagonicus (de Blainville, 1842), es un pequeño mustélido de distribución sudamericana, contando con registros para Argentina y Chile. Habita estepas herbáceas y arbustivas patagónicas de ambos países. Particularmente en Argentina, su distribución abarca desde la Patagonia hasta el sur de la provincia de Buenos Aires por el este, aunque por zonas áridas del oeste llega tan al norte como hasta la provincia de Salta, en estos sitios frecuenta ambientes áridos o semiáridos de matorrales o bosques xerófilos (Osgood 1943, Tamayo \& Frassinetti 1980, Prevosti \& Pardiñas 2001). A pesar de tan amplia distribución, la mayor cantidad de información proviene de hallazgos fósiles, colecciones de museos y unas pocas observaciones directas realizadas durante los siglos XIX y XX. Los datos actuales sobre su presencia, alcanzan tan solo 28 registros realizados desde 1950, de los cuales 12 corresponden a los últimos 15 años (Prevosti \& Pardiñas 2001, Teta et al. 2008, Prevosti et al. 2009, Díaz Isenrath et al. 2012, Formoso et al. 2016, Schiaffini 2017).

En el centro de Argentina, en las provincias de Córdoba y San Luis, la especie sólo se conoce por restos fósiles (Prevosti et al. 2009), mientras que existen registros más modernos para Santiago del Estero y La Pampa (Prevosti \& Pardiñas 2001, Formoso et al. 2016). Particularmente en la provincia de Córdoba, aunque es mencionado para la misma por Morando y Polop (1997) sin mayores detalles, el único registro fehaciente

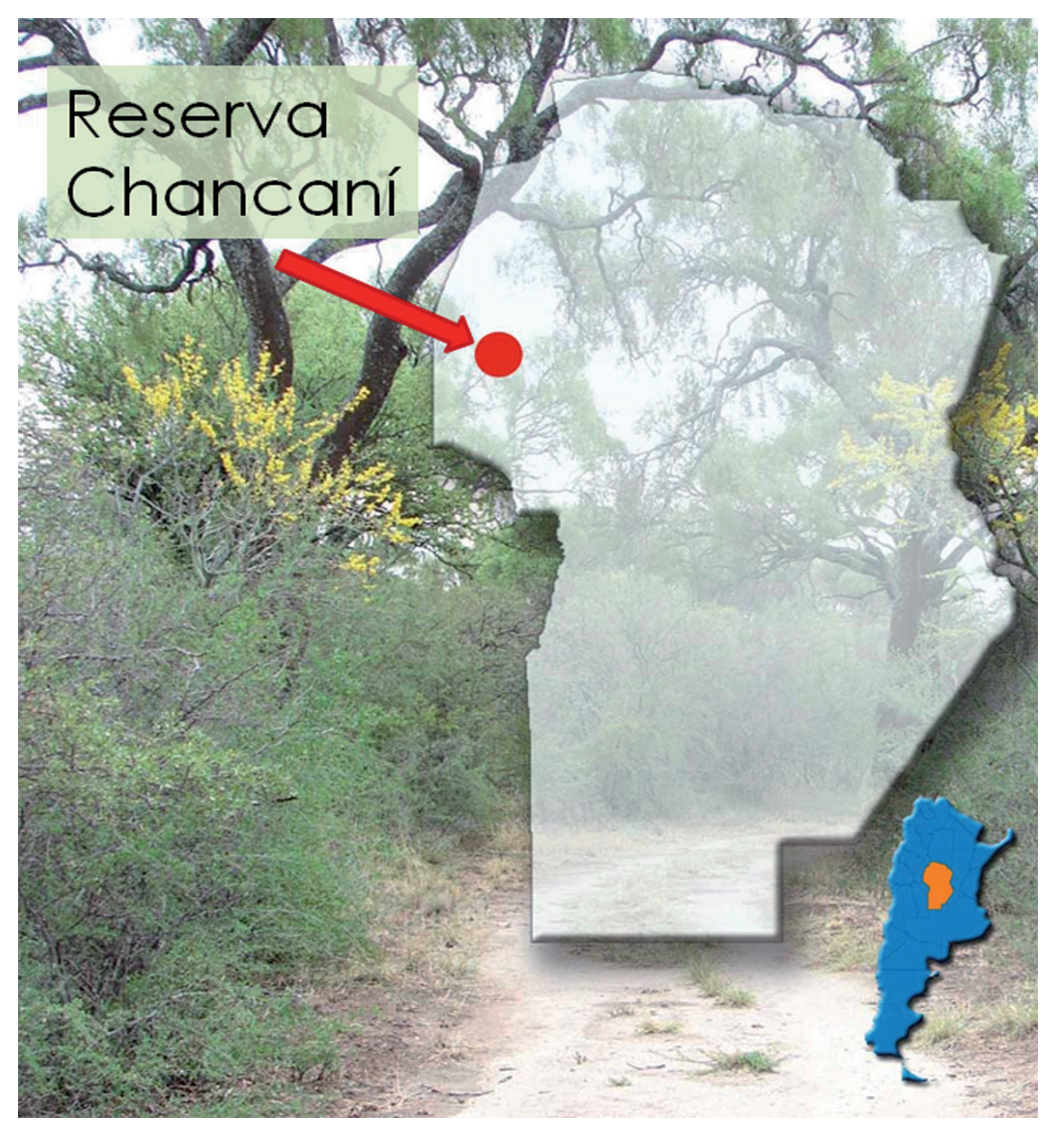

Figura 1. Ubicación geográfica de la Reserva Natural Chancaní, donde fue observado y fotografiado el ejemplar de Lyncodon patagonicus.

Figure 1. Geographical localization of the Natural Reserve Chanchaní, where the specimen of Lyncodon patagonicus was fotographied. 


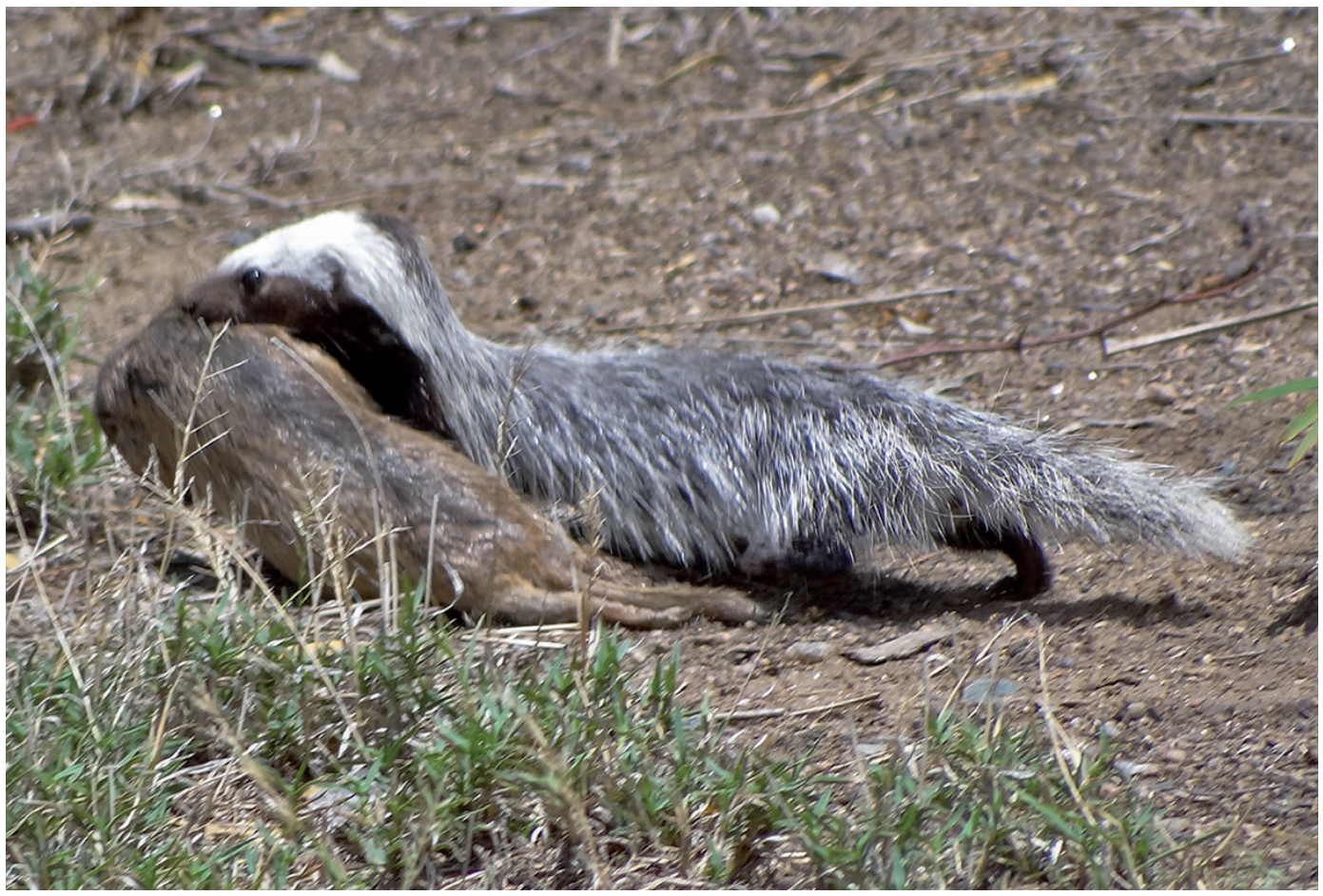

Figura 2. Ejemplar de Lyncodon Patagonicus llevando en su boca un individuo de Ctenomys sp. en la Reserva Natural Chancaní. 07 de diciembre de 2017. Foto: G. Sferco.

Figure 2. Specimen of Lyncodon Patagonicus carrying an individual of Ctenomys s.p. in the Natural reserve of Chancaní. December 7th, 2017. Photo: G. Sferco.

fue realizado por Florentino Ameghino en el departamento Capital, el cual data del Pleistoceno Tardío (Ameghino, 1889; Prevosti \& Pardiñas, 2001; Schiaffini et al., 2013). Además, Olrog (1976) señala a esta especie para Sol de Julio en la provincia de Santiago del Estero y áreas limítrofes de Córdoba; sin embargo, no existen ejemplares depositados en museos ni registros visuales provenientes de esta zona.

La historia natural de $L$. patagonicus también es muy poco conocida y la escasa información disponible es fragmentaria y basada en observaciones ocasionales (Cabrera y Yepes 1940, Redford y Eisenberg 1992). Dichas observaciones indican que la especie tiene actividad crepuscular y nocturna, y también sugieren que por su pequeño tamaño y otras evidencias indirectas su alimentación estaría basada fundamentalmente en roedores fosoriales (e.g. Ctenomys y Microcavia) y en aves (Koslowsky 1904, Cabrera y Yepes 1940).
En la presente comunicación se presenta el primer registro actual fidedigno de esta especie para la provincia de Córdoba, y se proporciona información complementaria sobre su biología.

Entre los días 7 y 8 de diciembre de 2017 se observó y fotografió un ejemplar de $L$. patagonicus en el Parque Provincial y Reserva Forestal Natural Chancaní $\left(31^{\circ} 22^{\prime} 32\right.$ "S

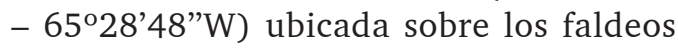
y llanuras vecinas de las sierras de PochoGuasapampa, hacia el oeste de la provincia de Córdoba, Argentina (Fig. 1). La reserva posee una superficie de casi 5000 ha y fitogeográficamente están representados los Distritos Serrano y Occidental de la Provincia Chaqueña (Cabrera 1976). Las observaciones corresponden a este último Distrito, cuya fisonomía es la de un bosque con predominio de quebracho blanco (Aspidosperma quebracho-blanco) en el estrato arbóreo, seguido en menor cantidad de algarrobo 


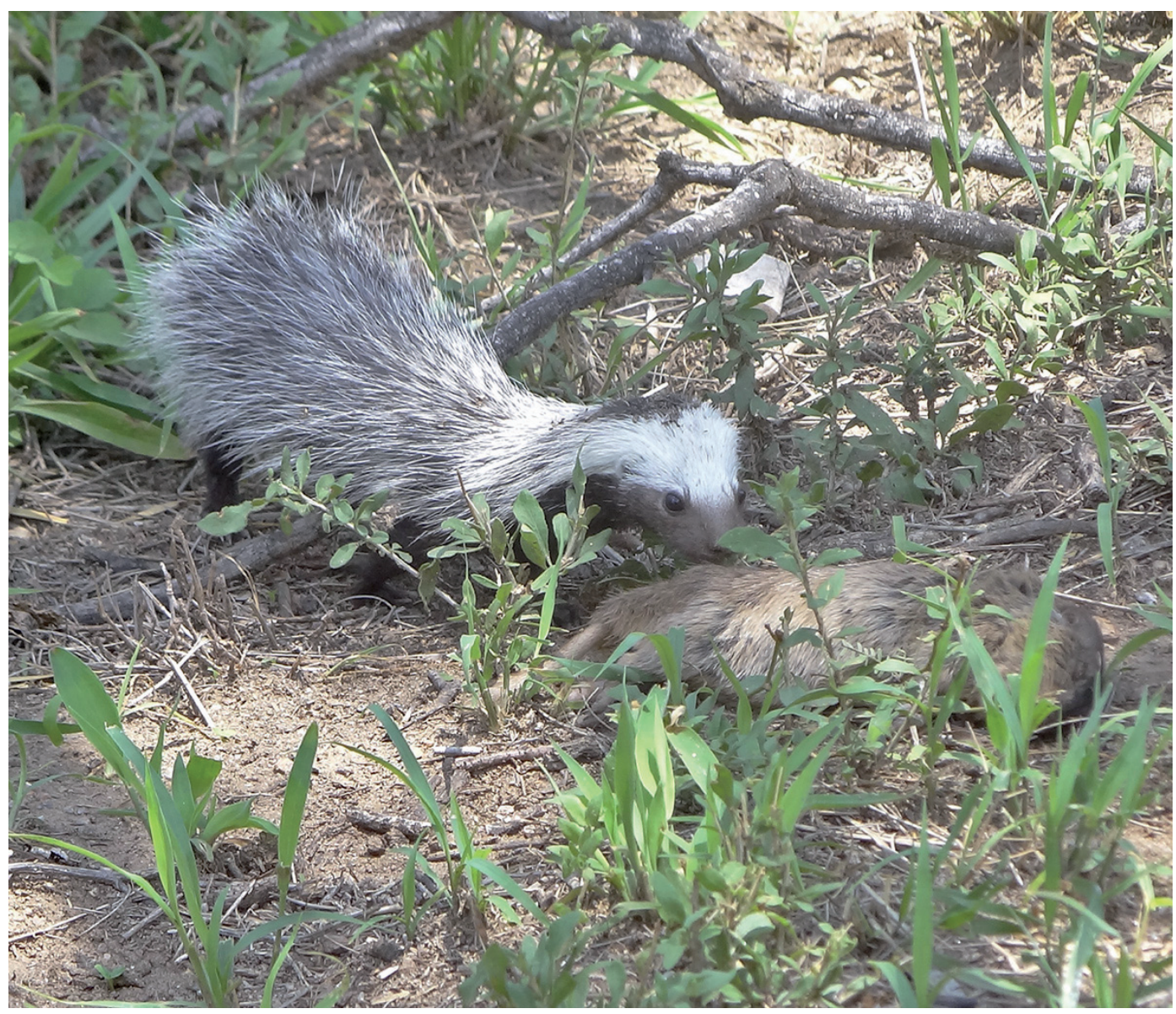

Figura 3. Ejemplar de Lyncodon patagonicus con el individuo de Ctenomys sp. a su lado en la Reserva Natural Chancaní. 07 de diciembre de 2017. Foto: G. Sferco.

Figure 3. Specimen of Lyncodon patagonicus with the individual of Ctenomys sp. to its side in the Natural reserve of Chanchani, December 7th. 2017.

(Prosopis spp.) y mistol (Ziziphus mistol). Mientras que los arbustos más representativos son: lata (Mimozyganthus carinatus), jarilla (Larrea divaricata) y garabato macho (Acacia furcatispina) (Carranza et al. 1992). El clima de la región pertenece al Dominio Semidesértico, con una temperatura media anual comprendida entre los $18^{\circ}$ y $23^{\circ} \mathrm{C}$ con un marcado déficit hídrico. Las precipitaciones oscilan entre los 350 y $500 \mathrm{~mm}$ anuales concentradas principalmente en primavera y verano (Capitanelli 1979).

El ejemplar de L. patagonicus fue detectado entre las 11 y las 16 hs durante dos días consecutivos en un sector de la Reserva destinado a la administración y zona de acampe de visitantes. El lugar presenta características típicas de parque, es decir que se ha eliminado el sotobosque predominando una vegetación herbácea y diferentes especies arbóreas, principalmente algarrobo y quebracho blanco, entremedio de las cuales se encuentran en forma aislada algunas madrigueras de Ctenomys. El primer día se lo vio recorriendo una de estas madrigueras, a la cual ingresaba y salía por una de sus entradas, que tenían un diámetro aproximado de entre 9 y $11 \mathrm{~cm}$. El segundo día fue observado y fotografiado (Figs. 2 y 3 ) en el mismo sector, en momentos en que llevaba en su boca un ejemplar de Ctenomys sp., el cual fue arrastrado por espacio de unos $15 \mathrm{~m}$ para luego introducirse con él en una ca- 
vidad en el suelo de $12 \mathrm{~cm}$, presumiblemente podría tratarse del ingreso a una madriguera de Ctenomys.

La identificación de la especie estuvo basada en su pequeño tamaño (el cual puede apreciarse en la Fig. 2 comparándolo con el individuo de Ctenomys), la ancha faja blanca frontal que se continúa hacia los flancos, la mancha negruzca de la nuca, el pequeño tamaño de las orejas y su pelaje largo (Pocock 1926; Cabrera \& Yepes 1940) (Fig. 3).

Nuestra observación fue realizada en una zona que Schiaffini (2017) señala como de aptitud media del hábitat. Además, confirmamos que al menos una parte de su dieta incluye roedores del género Ctenomys. Por otro lado, a pesar de que la información previa indica actividad crepuscular y nocturna para esta especie (Cabrera 1928, Cabrera \& Yepes 1940, Larivière \& Jennings 2009), nuestras observaciones coinciden con lo registrado por Formoso et al. (2016) en La Pampa, en cuanto a que también puede presentar actividad durante el día. Dado que la especie es considerada como cercana a la amenaza en Argentina (Díaz Isenrath et al. 2012), principalmente debido a que es eliminada por los habitantes rurales, resulta necesario realizar relevamientos dirigidos a verificar la presencia continua de la especie en el oeste de la provincia de Córdoba y en las vecinas provincias de San Luis, donde sólo se ha documentado su presencia por medio de restos fósiles y La Rioja, cuyo último registro data del año 1931 (Prevosti et al. 2009; Schiaffini 2017).

\section{AGRADECIMIENTOS}

A R. Torres por identificar el ejemplar y por la lectura crítica y sugerencias que mejoraron el manuscrito. G. Boaglio, S. Salvador y C. Rosacher también colaboraron en la identificación. A G. Haro por el préstamo de material bibliográfico. A dos revisores anónimos por sus sugerencias y comentarios.

\section{FINANCIAMIENTO}

La campaña realizada a la Reserva Chancaní, donde fueron realizadas las observacio- nes que derivaron en el presente manuscrito, no contó con subvención de ningún tipo. Las erogaciones fueron solventadas por cada uno de los autores.

\section{PARTICIPACIÓN}

M.T. fue quien detectó en primera instancia el ejemplar, y todos los autores tuvimos la posibilidad de observarlo. El segundo día, el ejemplar fue detectado por A.T. y A.G., G. S. y E. C. obtuvieron fotografías. Todos participamos en la búsqueda de antecedentes de la especie. El primer borrador lo realizó G. S., el cual fue revisado por el resto de los autores quienes aportaron sugerencias y modificaciones. La sección Bibliografía fue realizada por F. F. M. y F. R. Todos participamos en la edición final del manuscrito.

\section{CONFLICTO DE INTERÉS}

Los autores declaran que no existe conflicto de interés.

\section{LITERATURA CITADA}

Ameghino, F. (1889). Contribución al conocimiento de los mamíferos fósiles de la República Argentina. Actas de la Academia Nacional de Ciencias en Córdoba 6, 1-1027.

Cabrera, A. (1928). Sobre Lyncodon patagonicus con descripción de una nueva sub-especie. Revista Chilena de Historia Natural 32, 259263.

Cabrera, A. (1976). Regiones fitogeográficas argentinas. Enciclopedia argentina de agricultura y jardinería. Ed. Acme, Buenos Aires. 85 pp.

Cabrera, A. \& Yepes, J. (1940). Mamíferos Sud-Americanos (vida, costumbres y descripción]. Historia Natural Ediar, (370 p). Buenos Aires: Compañía Argentina de Editores.

Capitanelli. R. G. (1979). Clima. En: J. B. Vázquez, R. A. Miatello, \& M. E. Roqué (Eds.). Geografía física de la Provincia de Córdoba (pp. 45-138). Buenos Aires: Ed. Boldt.

Carranza, M., Cabido M., Acosta, A. \& Paez, S. (1992). Las comunidades vegetales del Parque Natural Provincial y Reserva Forestal Natural Chancaní, Provincia de Córdoba. Lilloa, 28, 7586.

Formoso, A., Dolsan, M., Sosa, R. A. \& Teta, 
P. [2016]. A new distributional record for Lyncodon patagonicus (Carnivora: Mustelidae], one of the smallest and least known mustelids of South America. Revista Mexicana de Biodiversidad, 87,1159 1161.

Díaz Isenrath, G., Aprile, G. \& Soler, L. (2012). Lyncodon patagonicus (de Blainville). En: Ojeda, R. A., V. Chillo y G. Díaz Isenrath (eds.). Libro Rojo de Mamíferos Amenazados de Argentina (pp. 107 108). Mendoza: SAREM.

Koslowsky, J. (1904). Dos mamíferos de Patagonia cazados en el valle del lago Blanco [Territorio del Chubut]. Revista del Museo de La Plata, 11, 129-132.

Larivière, S. \& Jennings., A. P. (2009). Family Mustelidae (weasels and relatives). En: D. E. Wilson \& R. A. Mittermeier [Eds.). Handbook of the Mammals of the World, (vol 1: Carnivores, pp.564 656). Barcelona: Lynx Editions.

Morando, M. \& Polop, J. J. (1997). Annotated checklist of mammal species of Córdoba province, Argentina. Mastozoología Neotropical, 4, 129136.

Olrog, C. C. (1976). Sobre mamíferos del noroeste argentino. Acta Zoológica Lilloana, 32, 5-14.

Osgood, W. H. (1943). The mammals of Chile. Field Museum of Natural History,

Zoological Series, 30, 1-268.

Pocock, R. I. (1926). The external characters of the Patagonian weasel (Lyncodon patagonicus]. Proceedings of the Zoological Society of London, 1926, pp. 1085-1094.
Prevosti, F. J. \& Pardiñas, U. F. J. (2001). Variaciones cronológicas de Lyncodon patagonicus (Carnivora: Mustelidae) durante el Cuaternario. Mastozoología Neotropical, 8, 2139.

Prevosti, F., Teta, P. \& Pardiñas, U. F. J. (2009). Distribution, natural history, and conservation of the Patagonian Weasel Lyncodon patagonicus. Small Carnivore Conservation, 41, 29-34.

Redford, K. H. \& Eisenberg, J. F. (1992). Mammals of the Neotropics: the Southern Cone. Chile, Argentina, Uruguay, Paraguay, (vol. 2, pp. 1 430). Chicago: The University of Chicago Press.

Schiaffini, M, Martin, G., Giménez, A. \& Prevosti, F. [2013). Distribution of Lyncodon patagonicus (Carnivora, Mustelidae): changes from the Last Glacial Maximum to the present. Journal of Mammalogy, 94[2]:339-350.

Schiaffini, M. I. (2017). Niche overlap and shared distributional patterns between two South American small carnivorans: Galictis cuja and Lyncodon patagonicus [Carnivora: Mustelidae]. Mammalia, 81, 455463.

Tamayo, M. \& Frassinetti, D. (1980). Catálogo de los mamíferos fósiles y vivientes de Chile. Boletín del Museo Nacional de Historia Natural (Chile) 37, 323399.

Teta, P., Prevosti, F. \& Trejo, A. (2008). Raptor predation and new locality records for the poorly known Patagonian Weasel [Lyncodon patagonicus] (Carnivora: Mustelidae. Mammalian Biology, 73,238 240. 